\title{
Perioperative Nursing for Adult Congenital Heart Disease with Severe Pulmonary Arterial Hypertension
}

\author{
Shunling Li ${ }^{1}$, Surui Liang ${ }^{2}$, Weihua Xue ${ }^{1}$ \\ ${ }^{1}$ The First Affiliated Hospital of Sun Yat-sen University, Guangzhou, China \\ ${ }^{2}$ Nethersole School of Nursing, Faculty of Medicine, Nethersole School of Nursing, Hong Kong, China \\ Email: Xueweihua7808@126.com
}

How to cite this paper: Li, S.L., Liang, S.R. and Xue, W.H. (2020) Perioperative Nursing for Adult Congenital Heart Disease with Severe Pulmonary Arterial Hypertension. International Journal of Clinical Medicine, 11, 538-547.

https://doi.org/10.4236/ijcm.2020.119046

Received: August 10, 2020

Accepted: September 18, 2020

Published: September 21, 2020

Copyright (C) 2020 by author(s) and Scientific Research Publishing Inc. This work is licensed under the Creative Commons Attribution International License (CC BY 4.0).

http://creativecommons.org/licenses/by/4.0/

\begin{abstract}
Objectives: To explore the main points of perioperative nursing for adult congenital heart disease with severe pulmonary arterial hypertension. Methods: A retrospective study of 13 patients with congenital heart disease and severe pulmonary arterial hypertension who admitted to the perioperative period of care from January 2018 to December 2019. To prevent perioperative complications of the patients, the focus is on respiratory and circulatory system care, followed by blood coagulation monitoring, digestive system protection and psychological care. Results: All 13 patients passed the perioperative period and were discharged from ICU. Conclusion: Adult congenital heart disease with severe pulmonary arterial hypertension has high perioperative risk, respiratory and circulatory system care is the key.
\end{abstract}

\section{Keywords}

Adult Congenital Heart Disease (ACHD), Pulmonary Arterial Hypertension (PAH), Perioperative Nursing

\section{Introduction}

Adult congenital heart disease (ACHD) covers a series of structural heart abnormalities that exist before birth, which belongs to the abnormal development of fetal heart [1]; Pulmonary arterial hypertension (PAH) is defined as average pulmonary arterial pressure $\geq 25 \mathrm{mmHg}(1 \mathrm{mmHg}=0.133 \mathrm{kPa})$, pulmonary arterial capillary wedge pressure $\leq 15 \mathrm{mmHg}$, and pulmonary vascular resistance index $>3 \mathrm{WU} / \mathrm{m}^{2}$ [2]. PAH is a common comorbidity of ACHD and it's a key factor in determining the indications and prognosis of ACHD surgery [3]. With 
the popularization of pulmonary vasodilator, the surgical indications for ACHD with severe PAH are relaxed, and new hope is seen in the treatment of such patients [4]. However, such patients still have a high risk of surgery, high perioperative mortality and high complications [5]. At present, there are many nursing researches on the complications of perioperative pulmonary hypertension crisis of such patients [6], because of its high fatality rate. The results of this study show that the pulmonary hypertension crisis has been well prevented, and the prognosis of such patients is good, so it is worth studying. However, the sample size of this study is only 13 cases, and there is no control group or comparison with other studies, so it is impossible to judge whether this is an improvement on previous work. According to the classification of echocardiography [7] (mild: 40 - $50 \mathrm{mmHg}$, moderate 50 - $80 \mathrm{mmHg}$ : severe $>80 \mathrm{mmHg}$ ). During the period from 2018.1.1 to 2019.12.1, a total of 144 ACHD were admitted to the extracardiac ICU of the hospital. 43 cases had no PAH, 64 cases were mild PAH, 24 cases were moderate $\mathrm{PAH}$, and 13 cases were within severe $\mathrm{PAH}$, all of them were operated on Treatment. This study is a summary paper of clinical experience, the perioperative care of patients with severe $\mathrm{PAH}$ is reported as follows.

\section{Objects and Methods}

\subsection{Objects}

There were 13 patients in this study, 5 males and 8 females, aged (33.92 \pm 15.32 , 22 - 68) years old. 6 cases of atrial septal defect, 3 cases of ventricular septal defect, 2 cases of patent ductus arteriosus, 1 case of partial endocardial cushion defect and atrial septal defect, 1 case of partial endocardial cushion defect and complete ventricular septal defect and the foramen ovale is patent. The obvious symptoms of all patients were chest tightness and shortness of breath after exercise. Some patients have cyanotic lips and swollen lower limbs. Preoperative echocardiography examination measured pulmonary artery systolic pressure 80 - $141 \mathrm{mmHg}(95.54 \pm 16.96 \mathrm{mmHg}, 1 \mathrm{mmHg}=0.133 \mathrm{kPa})$; right heart catheterization examination 70 - 130/33 - $65 \mathrm{mmHg}$. All 13 patients underwent open heart surgery under cardiopulmonary bypass, and the time of cardiopulmonary bypass was $2-7$ hours. This study is to consult medical records and there is almost no risk to the objects, so it has obtained the right of informed consent from the hospital's ethics committee.

\subsection{Methods}

\subsubsection{Respiratory Care}

Before surgery, give the patients low-flow oxygen $(1-2 \mathrm{~L} / \mathrm{min})$, three times a day, and $30 \mathrm{~min}$, in order to improve the hypoxic state of the whole body and improve lung reserve capacity; Let the patients master the breathing function training methods such as pursed lip breathing, deep breathing, effective coughing, and exercise breathing exerciser. After the operation, the patients are urged to practice the above methods, while turning over, patting the back, and getting 
out of bed as soon as possible to promote postoperative lung recruitment and prevent atelectasis.

Postoperative ventilator and sputum suction care: Suction as needed, auscultate the breath sounds of the lungs, observe the ventilator waveform, and turn over and pat the back before suctioning. If the patient is prone to restlessness, add sedatives appropriately before suctioning. Postoperative lung expansion and sputum suction are given to expand lung vesicles to prevent atelectasis. The ventilator is continuously heated and humidified. Q2h dumps the condensed water of the ventilator pipeline into the humidifier to prevent sudden disconnection of the ventilator pipeline from causing airway pressure fluctuations, alveolar collapse or irritation of the patient. The ventilator tube should be replaced once every 14 days, but if it is contaminated, replace it in time. General ventilator parameter mode selects BIPAP/SMIV; respiratory rate 16 - 20 times/min; PEEP 5 $8 \mathrm{mmHg}$; give proper hyperventilation, keep PACO2 at $30-35 \mathrm{mmHg}$, expand small pulmonary blood vessels; inhale oxygen concentration 35\% - 50\%, Maintain $\mathrm{PAO} 2 \geq 85 \mathrm{mmHg}$, avoid continuous high flow (>50\%) oxygen inhalation to prevent oxygen poisoning. Extubation care: According to the pulmonary artery pressure of the patients after the operation, the pulmonary artery pressure of 4 patients decreased significantly after the operation, and the catheter was extubated on the first day after the operation; other vital signs were stable, conscious and cooperating, good coughing ability during sputum suction, and extremities movement Good. One hour after the doctor reduced the parameters of the ventilator, the patient had no intolerance, and arterial blood gas analysis results were satisfactory, early extubation is recommended; After extubation, give budesonide suspension, compound ipratropium bromide solution, acetylcysteine solution and oxygen atomized inhalation for $15-20$ minutes to prevent throat edema, dilute sputum, and then oxygen atomize inhalation TID treatment; Supervise patients to cough and expectorate, while preparing tissues and garbage bags for them; Provide continuous warming and humidifying oxygen Treatment. All 13 patients were successfully extubated without any pulmonary hypertension crisis.

Prevent pulmonary infection: Strengthen nurses' hand hygiene; Raise the head of the patient's bed by about $30^{\circ}-45^{\circ}$; Promote closed aseptic sputum suction; Q8h monitor airbag pressure to maintain $25-30 \mathrm{mmHg}$; Subglottic suction every 2 hours; Oral care TID, sputum suction before care. Administer antibiotics regularly in accordance with the doctor's order to maintain the effective concentration of antibiotics, improve efficacy and avoid drug resistance. Follow the doctor's order to give immune globulin and thymopentin to improve immunity. Observe body temperature, nature of sputum, bedside chest radiograph, blood routine, etc. to track the treatment effect. In this study, 1 case of lung infection occurred, which was cured after strengthening antibiotics that changed cefoperazone sodium and sulbactam sodium to vancomycin hydrochloride and imipenem and cilastatin. 
Prevention of pleural effusion: Because of extracorporeal circulation and wound blood oozing, blood osmotic pressure is reduced, lung tissue fluid oozes. Infusion of red blood cells, plasma, etc. to supplement blood volume and coagulation factors according to doctor's order; routine infusion of human albumin; rehydration mainly with colloid to increase tissue osmotic pressure. Appropriate administration of diuretic, in the early postoperative period, under the condition of stable circulation, maintain the balance of daily intake and exit of patients about minus $500 \mathrm{ml} / \mathrm{h}$, maintain the "dry" state of lungs, and promote the recovery of patients. In this study, 2 cases of pleural effusion were corrected by pleural puncture and drainage, supplemented with colloid to increase osmotic pressure.

This study shows that such patients have the most pulmonary complications, so it is necessary to strengthen nursing care, promote recovery, and reduce hospital stay.

\subsubsection{Circulatory System Care}

Cardiotonic diuretic potassium supplements care: Patients have all right ventricular function decline before operation, and routine oral digoxin, spironolactone/furosemide tablets, potassium tablets, etc., slow down heart rate, reduce heart load and improve heart function before operation. When taking digoxin, monitor the heart rate and pulse, whether there are digestive tract reactions, etc., and check the digoxin concentration $(<2 \mathrm{ng} / \mathrm{ml})$ regularly to avoid digoxin overdose or poisoning. Spironolactone tablets are potassium-sparing diuretics, furosemide tablets are potassium-excretion diuretics, during the diuretic period we should regular blood gas analysis and prevent electrolyte disorders. In the early postoperative period, the patient's heart is in a period of depression, and cedilan, dobutamine, norepinephrine, and dopamine cardiotonic drugs are given as directed by the doctor. When BNP continues to rise, and other cardiotonic drugs are not effective, they are generally given levosimendan then maintains blood gas calcium ions $1.25-1.35 \mathrm{~mol} / \mathrm{L}$ [8]. Dobutamine is recommended, a small dose ( $1-5 \mathrm{ug} / \mathrm{kg} / \mathrm{min})$ can positively improve muscle strength and vasodilation, and improve myocardial oxygen supply; The dose of norepinephrine should not exceed $2 \mathrm{ug} / \mathrm{kg} / \mathrm{min}$, and vasoconstriction It is easy to lead to insufficient renal perfusion, peripheral ischemia and hypoxia; When the single drug dose is too large, it is recommended to use the drug together. If necessary, give 654-2 to dilate the peripheral blood vessels according to the doctor's order; Ensure that the blood potassium and calcium are normal before using cedilan, calcium supplement at least 2 hours apart; Follow the doctor's order to supplement potassium magnesium aspartate or physiological saline $30 \mathrm{ml}$ and potassium chloride $20 \mathrm{ml}$ or magnesium sulfate $10 \mathrm{ml}$ and potassium chloride $20 \mathrm{ml}$ according to urine output and the degree of potassium deficiency at $5-30 \mathrm{ml} / \mathrm{h}$. Follow the doctor's order for diuresis of furosemide and torasemide, the hourly urine output is maintained at about $90-120 \mathrm{ml}$, it is forbidden to have excessive diuresis at one time, causing insufficient blood volume and inducing heart fail- 
ure. During ICU stay, an arterial blood gas analysis is done every 1 - 4 hours according to the condition and arterial blood gas results to keep acid-base and electrolyte stable, after transfer from the ICU, the medication was gradually changed to oral, similar to preoperative.

Vital signs and intake-output monitoring: to maintain a stable vital sign and avoid large fluctuations. CVP 5 - $12 \mathrm{mmHg}$; heart rate 60 - 110 beats/min; MBP maintained above $65 \mathrm{mmHg}$; body temperature above $36.3^{\circ} \mathrm{C}$, and some patients whose body temperature is lower than $36^{\circ} \mathrm{C}$ after operation should be kept warm by air heater. If the vital signs are abnormal, report to the doctor in time, check the cause, deal with it in time, and track the effect to prevent serious complications. Patients with decreased heart function, too much or too little intake in a short time can induce acute heart failure, usually the infusion rate is less than $100 \mathrm{ml} / \mathrm{h}$. Such patients have more blood or blood products after surgery, also need to control the speed, and timely diuresis.

\subsubsection{Nursing Vasodilator}

General vasodilator care: Milrinone's positive muscle strength and vasodilator effects can synergistically reduce pulmonary artery pressure; It is administered for short-term postoperatively, maintaining $0.25-1.0 \mu \mathrm{g} / \mathrm{kg} / \mathrm{min}$; Overdose may cause hypotension and tachycardia. In the case of high blood pressure, add urapidil or nitroglycerin at the same time. Urapidil inhibits peripheral vasoconstriction and reduces vascular resistance and cardiac load. It can be directly injected slowly with 10 - $50 \mathrm{mg}$ intravenously. The effect is usually displayed within 5 minutes, and subsequent intravenous pumping can be continued; note that a sudden drop in blood pressure can cause bradycardia or even the heart sudden stop. Nitroglycerin reduces vascular smooth muscle tension, reduces venous return heart blood volume, peripheral vascular resistance reduces cardiac filling pressure and reduces myocardial oxygen consumption, while redistributing blood flow along the collateral vascular bed from epicardium to endocardium to improve myocardial supply Oxygen; note that it may cause headaches, nausea, hypotension and tachycardia. Antihypertensive drugs require separate access to prevent drastic fluctuations in blood pressure and pulmonary artery pressure caused by infusion or intravenous bolus drugs.

Pulmonary vasodilator care [9]: 1) Prostacyclins (treprostinil, iloprost) can relax blood vessels, protect vascular endothelial cells, prevent vascular smooth muscle cell proliferation, inhibit platelet aggregation and other functions. 2) Phosphodiesterase-5 (PDE-5) inhibitors (sildenafil, tadalafil) can inhibit the decomposition of cyclic guanylic acid, increase NO content, relax smooth muscles, and reduce pulmonary blood pressure. 3) Endothelin receptor antagonists (bosentan, ambrisentan) can reduce pulmonary vascular resistance, reconstruct pulmonary blood vessels, reverse right ventricular hypertrophy, and improve Patient exercise tolerance and quality of life. 4) Due to the inconvenience of inhalation and adjustment of the micro-flow of NO gas and the easy leakage of the gas, it is less used in our department. In this study, only 1 patient was used in 
combination with other drugs when it was very difficult to control postoperative PAH.

At present, the evidence is relatively sufficient that the combination of prostacyclins and PDE-5 inhibitors is safe and effective. Whether other combination therapies are effective is still controversial [10] [11].

The most commonly used drug combination in this study: Sildenafil or Tadalafil and Treprostinil. Treprostinil is diluted with sterile water for injection or $0.9 \% \mathrm{NaCl}$ solution and then pumped into the vein, or injected subcutaneously with pure solution. Subcutaneous injections are generally used before surgery. Intravenous pumping during surgery is helpful to quickly control the fluctuation of PAH. After surgery, intravenous pumping is gradually changed to subcutaneous injection. Patients with intravenous pumping are well tolerated. Common adverse reactions of subcutaneous injection are pain at the injection site (usually starting at 12 hours, local pain within 2 - 7 days), erythema, rash and sclerosis, etc.; But the symptoms can generally be relieved after treatment by changing the injection site, oral analgesics, and topical application of mucopolysaccharide polysulfate cream, etc.; Other adverse reactions include diarrhea, jaw pain, edema, hypotension, dizziness, nausea, vomiting, etc.; Symptoms generally disappear after reducing the drug dose and symptomatic treatment. In this study, 2 patients developed nausea and vomiting, and the symptoms were relieved after the dose was reduced. Treprostinil can be safely used in infants and young children. Cidirafil has a half-life of 4 hours, orally three times a day, usually $25 \mathrm{mg}$ each time, common adverse reactions include headache, flushing, and indigestion. Tadalafil has a half-life of $17.5 \mathrm{~h}$. It is taken orally twice a day, usually $5 \mathrm{mg}$ each time, common adverse reactions include headache and indigestion. There is a risk of hypotension when PDE-5 inhibitors are used together with antihypertensive drugs. Bosentan has a half-life of 5.4 hours and is taken orally twice a day, usually $62.5 \mathrm{mg}$ each time, common adverse reactions include abnormal liver function, anemia, fluid retention, headaches, etc., during the period of medication, liver function needs to be monitored monthly. Ambrisentan has a half-life of 9 hours. It is taken orally twice a day, usually $5 \mathrm{mg}$ each time, common adverse reactions include anemia, headache, palpitations, hot flashes, nasal congestion, fluid retention, etc. The increase in liver enzymes is not significant, and there is no need to closely monitor liver function, Have better safety and tolerance.

\subsubsection{Sedative and Analgesic Care}

Pain and restlessness cause body stress, increase organ metabolism and aggravate the condition. Diazepam tablets $2.5 \mathrm{mg}$ - $5 \mathrm{mg}$ were given the night before surgery to ensure the quality of sleep before surgery. Patients are generally given remifentanil and dexmedetomidine continuous intravenous pumping in the ICU after surgery, and midazolam is generally used for intravenous bolus injection when the patient is suddenly irritable. Adverse reactions of remifentanil include nausea, vomiting, respiratory depression, bradycardia, and hypotension. Adverse 
reactions of dexmedetomidine include hypotension and slow heart rate. Excessive use of sedative analgesics will prolong the time of mechanical ventilation, hospital stay and even mortality. Therefore, the use of drugs should be minimized when the requirements of sedative pain are met. The patient returned to the ICU after the operation, and the surgeon explained the patient's condition and gave advice on the time of extubation for tracheal intubation. If the postoperative PAH drops significantly and the condition is relatively stable, the tube is usually extubated on the first day after surgery.

Deep sedation (RASS -3 to -5 points) if there is still severe PAH and unstable organ function after the operation. Light sedation (RASS -2 to 1 points) when organ function is relatively stable and pulmonary artery pressure is well controlled. When the tracheal intubation needs to be removed, only low-dose dexmedetomidine is used for continuous intravenous pumping, which has a good analgesic effect. Analgesia goals: Patients who can express themselves use NRS score < 4 points; patients who cannot express, but have good motor function and behavior can be observed, use CPOT $<3$ points [12]. After returning to the general ward, the patient was given diazepam $2.5 \mathrm{mg}$ at night to ensure the quality of postoperative sleep. Encourage patients to get out of bed and exercise. According to the wound pain, fentanyl transdermal patch and indomethacin orally are given symptomatically.

\subsubsection{Nursing of Coagulation System, Digestive System, Nervous System and Urinary System}

Heparinization of cardiopulmonary bypass, destruction of coagulation factors and platelets, bleeding, all patients had coagulation disorders after operation. Protamine was given to neutralize heparin after surgery to maintain activated whole blood clotting time (ACT) < 120 seconds; Generally, hemocoagulase and tranexamic acid BID be used only one time after surgery. Hourly record the volume, color and nature of pericardial mediastinum or chest drainage fluid; Squeeze the pericardial mediastinal drainage tube every hour; Continuous low-pressure suction (not necessary for thoracic drainage tube) to keep drainage smooth. If the drainage volume exceeds $100 \mathrm{ml}$ per hour, it lasts for 2 hours and the color is bright red must be reported to the doctor. According to the drainage volume, blood routine examination and coagulation routine, follow the doctor's order to give hemostatic drugs, plasma, cryoprecipitate, etc. In this study, there was one case of active bleeding. The coagulation routine was detected immediately, and the symptoms were controlled after hemostatic treatment was given. There was one case of heparin-induced thrombocytopenia, observe whether there are bleeding symptoms throughout the body, pay attention to mild strength during nursing; The effect of giving platelet-increasing drugs and platelet transfusion was not good; Later, the patient was transferred to a local medical institution to continue treatment, then in the ICU for 1 month and transferred to the general ward for 1 month, now the patient living well.

Low perfusion during cardiopulmonary bypass, too long preoperative fasting, 
and the influence of various drugs on the digestive system. On the first postoperative day, patients with no planned extubation should be left with a gastric tube. Live combined was given to adjust the intestinal flora; $5 \%$ glucose solution and enteral nutrition solution were pumped at a low flow rate $(10 \mathrm{ml} / \mathrm{h})$, dilute the enteral nutrient solution, reduce the incidence of diarrhea, and reduce gastrointestinal function damage. The gastric tube is fixed by the high platform method, and the gastric tube is withdrawn every shift to avoid gastric retention, aspiration and pneumonia. Regularly check liver function and blood routine, routinely give stomach medicine and adequate nutrition after operation. Observe for gastrointestinal bleeding, abdominal pain, diarrhea, etc. At present, the latest care guidelines for peripheral cardiac surgery encourage oral carbohydrate beverages 2 to 4 hours before surgery, which can reduce postoperative insulin resistance and tissue glycosylation, improve postoperative blood glucose control, and enhance the recovery of intestinal function [13].

With atrial fibrillation and coagulation disorders, patients are at risk of cerebral hemorrhage or stroke after surgery. Each class monitors pupils, consciousness, and limb muscle strength. For patients with deep sedation, gradually reduce the sedatives in the morning and increase the dose after the evaluation. Especially for patients who have a long operation time and do not wake up within 4 hours after surgery, close assessment and timely report.

Cardiopulmonary bypass destroys red blood cells, hypoperfusion, acidosis, and some drugs affect renal function. Patients may have acute kidney injury after surgery. Record hourly urine output $(>1 \mathrm{ml} / \mathrm{kg} / \mathrm{h}$ ), color and nature, and regularly check renal function and urine routine. If there is any abnormality, report in time, give diuretic according to doctor's order, and do bedside CRRT treatment to prevent renal failure.

\subsubsection{Psychological Care}

Keeping the patient's mood stable and increasing the psychological defense function have a positive effect on the prognosis. ICU nurses make preoperative visits, introduce the ICU environment, explain the discomfort that may occur after the operation, and the corresponding sign language expressions, encourage patients to overcome temporary difficulties, and adjust their mentality to treat the critical postoperative period. Dynamic nursing: 1) Psychological support: ask patients about their condition and surgery questions or worries before surgery, give targeted answers, and encourage patients who recover after surgery to answer them to improve the effect. Give notification before any nursing operation, explain the condition appropriately every day, always comfort and encourage. The target group includes patients and caregivers. 2) Psychological relaxation: Explain the importance of emotional stability in promoting recovery. Ask the patient how he overcomes mood swings and deliberately practice these skills before surgery. For example, prepare the radio, encourage recordings from family members, calm songs, listen to the recitation of Buddhist scriptures, etc., take a slow and deep breath to relax the whole body and calm the mood. 


\section{Results}

Complications: 1 case of pulmonary infection, 2 cases of pleural effusion, 1 case of atelectasis, 1 case of heparin-induced thrombocytopenia, 1 case of active bleeding, all were corrected after symptomatic treatment. All 13 patients passed the perioperative period. One patient with heparin-induced thrombocytopenia was temporarily incurable, and was discharged smoothly after being transferred to a local hospital for continued treatment at the request of his family. The average number of days of hospitalization before surgery $(13.15 \pm 6.84)$, the average number of days of hospitalization in ICU after surgery $(4.17 \pm 2.23)$, the average number of days of tracheal intubation $(2.92 \pm 1.8)$, and the average number of days of hospitalization in general wards after surgery $(13.54 \pm 7.87)$.

\section{Conclusion}

With the application of lung-targeted antihypertensive drugs, the scope of surgery for patients with severe $\mathrm{PAH}$ has been expanded. Although patients are at high perioperative risk, especially pulmonary hypertension crisis is one of the important causes of perioperative death, effective sedation Treatment of pain relief and reduction of PAH, pulmonary hypertension crisis can be well prevented. After better and better care and treatment, the postoperative results of adult congenital heart disease with severe PAH are worth looking forward to.

\section{Limitations}

This study is a descriptive study that summarizes the main points of perioperative care and there is no control group. The sample size is small. The long-term effect of the patient was not followed after discharge.

\section{Conflicts of Interest}

The authors declare no conflicts of interest regarding the publication of this paper.

\section{References}

[1] Stout, K.K., Daniels, C.J., Aboulhosn, J.A., Bozkurt, B., Broberg, C.S., Colman, J.M. and Van Hare, G.F. (2019) 2018 AHA/ACC Guideline for the Management of Adults with Congenital Heart Disease: Executive Summary: A Report of the American College of Cardiology/American Heart Association Task Force on Clinical Practice Guidelines. Circulation, 139, e637-e697.

https://doi.org/10.1161/CIR.0000000000000602

[2] Galie, N., Humbert, M., Vachiery, J.L., Gibbs, S., Lang, I., Torbicki, A. and Hoeper, M. (2016) 2015 ESC/ERS Guidelines for the Diagnosis and Treatment of Pulmonary Hypertension: The Joint Task Force for the Diagnosis and Treatment of Pulmonary Hypertension of the European Society of Cardiology (ESC) and the European Respiratory Society (ERS): Endorsed by: Association for European Paediatric and Congenital Cardiology (AEPC), International Society for Heart and Lung Transplantation (ISHLT). European Heart Journal, 37, 67-119.

https://doi.org/10.1093/eurheartj/ehv317 
[3] Roth, T.S. and Aboulhosn, J.A. (2016) Pulmonary Hypertension and Congenital Heart Disease. Cardiology Clinics, 34, 391-400. https://doi.org/10.1016/j.ccl.2016.04.002

[4] Jing, X.Y., Yang, X.Y., Su, J.W., Liu, C.H., Chen, Z., Gu, H. and Liu, Y.L. (2016) Study of Diagnostic Treatment in Patients Suffering Congenital Heart Disease Combined with Severe Pulmonary Arterial Hypertension. Journal of Cardiovascular and Pulmonary Diseases, 35, 293-296.

[5] Schuijt, M., Blok, I.M., Zwinderman, A.H., van Riel, A., Schuuring, M.J., de Winter, R.J. and Bouma, B.J. (2017) Mortality in Pulmonary Arterial Hypertension Due to Congenital Heart Disease: Serial Changes Improve Prognostication. International Journal of Cardiology, 243, 449-453. https://doi.org/10.1016/j.ijcard.2017.05.101

[6] Zhang, H.H., Wang, L.L., Yu, C.Q., Xing, X.M., Zhi, W.C., Lyu, L. and Feng, H.Y. (2018) Prevention and Treatment of Perioperative Pulmonary Hypertensive Crisis in Patients with Serious Pulmonary Arterial Hypertension Relevant with Ventricular Septal Defect. Chinese Journal of Practical Nursing, 34, 705-708.

[7] Hatle, L., Angelsen, B.A. and Tromsdal, A. (1981) Non-Invasive Estimation of Pulmonary Artery Systolic Pressure with Doppler Ultrasound. BMJ Journals, 45, 157-165. https://doi.org/10.1136/hrt.45.2.157

[8] Jiang, R., Zhao, Q.H., Wu, W.H., Zhang, R., Yuan, P., Gong, S.G. and Liu, J.M. (2018) Efficacy and Safety of a Calcium Sensitizer, Levosimendan, in Patients with Right Heart Failure Due to Pulmonary Hypertension. Clinical Respiratory Journal, 12, 1518-1525. https://doi.org/10.1111/crj.12699

[9] van Dissel, A.C., Mulder, B.J. and Bouma, B.J. (2017) The Changing Landscape of Pulmonary Arterial Hypertension in the Adult with Congenital Heart Disease. Journal of Clinical Medicine, 6, 40. https://doi.org/10.3390/jcm6040040

[10] Zhang, C.H.Y.H. (2014) Effects of Iloprost Combined with Low Dose Tadalafil in Adult Congenital Heart Disease Patients with Severe Pulmonary Arterial Hypertension: A Single-Center, Open-Label Controlled Study. Chinese Journal of Cardiology, 42, 474-480.

[11] Simonneau, G., Rubin, L.J., Galie, N., Barst, R.J., Fleming, T.R., Frost, A. and Badesch, D.B. (2014) Long-Term Sildenafil Added to Intravenous Epoprostenol in $\mathrm{Pa}$ tients with Pulmonary Arterial Hypertension. The Journal of Heart and Lung Transplantation, 33, 689-697. https://doi.org/10.1016/j.healun.2014.02.019

[12] Association, C.C.M.B. (2018) Guidelines for Analgesia and Sedation Treatment in Intensive Care Unit of Chinese Adults. Chinese Critical Care Medicine, 30, 497-514.

[13] Engelman, D.T., Ben, A.W., Williams, J.B., Perrault, L.P., Reddy, V.S., Arora, R.C. and Boyle, E.M. (2019) Guidelines for Perioperative Care in Cardiac Surgery: Enhanced Recovery after Surgery Society Recommendations. JAMA Surgery, 154, 755-766. https://doi.org/10.1001/jamasurg.2019.1153 